\title{
Entrenamiento de balonmano en las categorías junior y senior: diferencias y similitudes
}

\section{Handball training in $\mathrm{u}-21$ and adult teams: differences and similarities}

\author{
Rafael Pombo Menezes ${ }^{1 *}$, Benjamín Longarela Pérez ${ }^{2}$ y Larissa Rafaela Galatti ${ }^{3}$ \\ 1 Universidade de São Paulo (USP). Escola de Educação Física e Esporte de Ribeirão Preto (EEFERP) (Brasil). \\ 2 Facultad de Ciencias del Deporte y la Actividad Física, Universidade da Coruña (España). \\ 3 Faculdade de Ciências Aplicadas, Universidade Estadual de Campinas, Limeira, SP (Brasil).
}

\begin{abstract}
Resumen: Entrenadores de balonmano buscan el desarrollo de diferentes capacidades de los jugadores, a través de diferentes métodos de enseñanza. El objetivo del presente estudio fue identificar las diferencias y similitudes metodológicas en el proceso de entrenamiento de las categorías júnior y sénior. Se entrevistaron seis entrenadores de balonmano y las entrevistas fueron analizadas con el método Discurso del Sujeto Colectivo. Los entrenadores mencionaron características similares en las categorías: el aumento de la especificidad y la obtención de resultados a partir de la categoría júnior; y como principales diferencias: las experiencias competitivas y la búsqueda de resultados. Los entrenadores señalaron que utilizan métodos de enseńanza similares en ambas categorías, principalmente por entrenaren en conjunto y por la necesidad de obtener resultados. Aunque los entrenadores creen que es necesario que las jugadoras tengan más experiencias relacionadas con el ambiente competitivo, hay un reconocimiento hacia los contenidos con los que tuvieron contacto durante la formación deportiva.

Palabras clave: Entrenador deportivo; Pedagogía del deporte; Entrenamiento deportivo; Balonmano; Deporte femenino.
\end{abstract}

Abstract: Handball coaches aim to develop different players' skills, using different teaching approaches. The aim of this work was to identify the differences and methodological proximity between the junior and adult teams of women's handball. Six handball coaches were interviewed and the speeches were analyzed using the Collective Subject Discourse method. The coaches mentioned similar characteristics of both teams, and point out the increase in specificity and results collection from the junior team; and the main differences between these teams are related to the competitive experiences and the magnitude of the results charged. Coaches have been using similar teaching approaches mainly because the integration between the teams and the search for expressive results in competitions. Although coaches believe that there is a need for more competitive experiences for women players, there is a charge for the content with which they are supposed to have contact throughout their entire training process.

Keywords: Sport coaching; Sport pedagogy; Sport training; Handball; Women's handball.

\section{Introducción}

La naturaleza compleja del balonmano está directamente influenciada por las relaciones de cooperación y oposición entre los jugadores (Menezes, 2012). En su contexto se pretende constantemente la posesión del balón, a partir de una disputa simultánea en espacios comunes, intentando meter gol o evitar que el adversario anote.

El proceso de enseńanza-aprendizaje de los juegos deportivos se produce a largo plazo, englobando diferentes elementos (técnico-tácticos y de preparación física) y desmandando estrategias para que los jugadores puedan desarrollarse paulatinamente (Antón García, 1990; Ehret, Späte, Schubert y Roth, 2002). A lo largo de este proceso, cabe señalar que se produce un incremento en las exigencias de aspectos como la preparación física y los niveles de especialización de los jugadores, a medida que se aproximan a la edad adulta.

Entendiendo el proceso de enseńanza-aprendizaje como un proceso a largo plazo y aunque los jugadores presentan diferentes demandas específicas, Durand-Bush y Salmela (2002) consideran cuatro etapas para la formación de los

Dirección para correspondencia [Correspondence address]: Rafael Pombo Menezes. Email: rafaelpombo@usp.br jugadores: diversificación (ocurre en los primero años, con los primeros contactos con el deporte); especialización (engloba desde el inicio de la práctica de la modalidad escogida hasta la definición de la misma como carrera deportiva); inversión (referente a los años dedicados a la carrera deportiva); y manutención (equivale a los años de éxitos deportivos, cuando los jugadores obtienen sus mejores resultados y se preocupan no solamente por mantenerlos, sino también por aumentarlos).

En el modelo propuesto por Côtè, Baker y Abernethy (2007) se señalan diferentes etapas para la formación de jugadores, estableciéndose inicialmente actividades generalistas (juegos deliberados), orientándose a lo largo del proceso, hacia actividades más específicas (prácticas deliberadas), apuntando hacia el desarrollo deportivo, culminando con la especialización deportiva (alrededor de los 16 años, etapa de inversión). No obstante, Côtè et al. (2007) y Galatti, Reverdito, Scaglia, Paes y Seoane (2014) señalan como relevante la diversificación a lo largo del proceso de formación de los jugadores, identificando la especialización y perfeccionamiento técnico-táctico a partir de los 15 ańos de edad (etapa de inversión), prologándose esta etapa hasta el inicio de la fase adulta 
siendo fundamental para la siguiente etapa, de manutención (Durand-Bush y Salmela, 2002).

En relación al balonmano, diferentes autores presentan propuestas para el desarrollo de los jugadores a largo plazo (Ehret et al., 2002; Greco, Silva y Greco, 2012) y otros que identificaron aspectos de diferentes categorías por medio de la opinión de los entrenadores (Menezes, Marques y Nunomura, 2015, 2017). La familiarización con los contenidos, proporcionada por los diferentes métodos de enseñanza, es necesaria para la comprensión del juego y del ambiente competitivo, lo que ocurre gradualmente, en la medida en que los jugadores completan cada etapa de este proceso (Menezes, Reis y Tourinho Filho, 2015).

La categoría U-16 es señalada como el inicio de la etapa de especialización, considerada muy importante en el proceso de formación de los jugadores (Baratti y Casali, 2008; Ehret et al., 2002), acentuándose en la categoría júnior y obteniendo su ápice en la categoría sénior. A los 18 años, los jugadores que ya están inmersos en un proceso a largo plazo se encuentran en la fase de inversión, en la cual se busca un mayor rendimiento y hay mayor espacio para prácticas especializadas de la modalidad (Côtè et al., 2007). En ese sentido, cabe destacar que los jugadores de balonmano tienden a presentar mejores índices de desempeño entre los 22 y 26 años (Bompa y Haff, 2009).

Comprender los aspectos inherentes al proceso de formación deportiva a largo plazo permite planificar la distribución de los contenidos hasta la categoría adulta, teniendo en cuenta los cambios que ocurren en relación a las competiciones y a los aspectos de crecimiento y maduración. Estas premisas pueden evitar prácticas que promuevan la especialización deportiva precoz, que tan criticada ha sido por diferentes autores (Côtè et al., 2007).

Teniendo en cuenta el contexto presentado, este estudio transcurre entre el final de la etapa de inversión y el inicio de la etapa de manutención de jugadoras de balonmano. De este modo, el objetivo fue identificar, desde la perspectiva de los entrenadores, las diferencias y similitudes metodológicas en el proceso de enseńanza-aprendizaje de las categorías júnior y sénior.

\section{Método}

Participaron en el presente estudio seis entrenadores de balonmano (S1, S2, S3, S4, S5, S6) que cumplían con los siguientes requisitos para ser incluidos: a) ser entrenador de equipos femeninos de categoría sénior y estar en activo en las fechas en las que se recogieron los datos; b) haber clasificado a su equipo sénior entre las tres primeras, en la primera y segunda división, en los Juegos Abiertos del Interior del Estado de São Paulo; c) haber ejercido como entrenador de todas las categorías de formación.
La decisión de seleccionar el Estado de São Paulo viene determinada por destacar como escenario del balonmano nacional (Menezes, Marques y Morato, 2016), así como por la proximidad entre los investigadores y los entrenadores. Para recoger la opinión de los entrenadores deportivos, este estudio fue evaluado y aprobado por un Comité de Ética en Investigación Institucional (411.338), de modo que los participantes firmaron un Término de Consentimiento Libre y Esclarecido (TCLE), el cual garantiza la confidencialidad de los datos personales y el uso de las informaciones exclusivamente para fines académicos. Las características de los participantes se presentan en la tabla 1.

Tabla 1: Datos de los entrenadores.

\begin{tabular}{ccccc}
\hline Entrenador & Edad & $\begin{array}{c}\text { Experiencia como } \\
\text { entrenador }\end{array}$ & $\begin{array}{c}\text { Graduado } \\
\text { en EF }\end{array}$ & $\begin{array}{c}\text { Ańos como } \\
\text { graduado }\end{array}$ \\
\hline S1 & 49 & 28 & $\mathrm{Si}$ & 28 \\
S2 & 48 & 13 & $\mathrm{Si}$ & 28 \\
S3 & 42 & 20 & $\mathrm{Si}$ & 19 \\
S4 & 31 & 13 & $\mathrm{Si}$ & 10 \\
S5 & 42 & 14 & $\mathrm{Si}$ & 8 \\
S6 & 44 & 20 & $\mathrm{Si}$ & 15 \\
\hline
\end{tabular}

Los participantes tenían una media de edad de 42.7 años ( \pm 6.4 ; mín=31 y máx=49), con un periodo medio de actuación profesional de 18.0 años ( \pm 5.9 ; mín=13 y máx=28). Todos los entrenadores son graduados en Educación Física (con una media de $18.0 \pm 8.6$ ańos como graduados), teniendo en cuenta que tres de ellos completaron un curso de especialización en áreas relacionadas con el balonmano.

La decisión de realizar una investigación cualitativa viene determinada por la temática, para poder envolver aspectos complejos del comportamiento humano, de los cuales se pretenden análisis más detallados (Marconi y Lakatos, 2011), en especial sobre el proceso de enseńanza-aprendizaje en las categorías júnior y sénior del balonmano. De este modo, se pretendió acceder a información de naturaleza descriptiva, orientando la atención del investigador hacia los significados atribuidos a los hechos y a los procesos (Triviños, 1987).

En lo que se refiere a la naturaleza de las informaciones para ser identificadas y analizadas, y teniendo en cuenta los tipos de herramientas para la producción de datos en investigación cualitativa, se desarrolló un instrumento amplio de entrevista semiestructurada, compuesto por dos bloques: a) información personal y formación académica; b) contenidos y métodos de enseñanza en diferentes categorías. Fueron seleccionados los discursos englobados en el bloque "b" que se referían especialmente a las categorías júnior y sénior. Las cuestiones principales fueron: "hable sobre las actividades/ejercicios en la categoría júnior"; "hable sobre las actividades/ejercicios en la categoría adulta”. A partir del tratamiento de los resultados obtenidos, 
fue posible identificar un panorama sobre los aspectos envueltos en el entrenamiento de esas categorías.

Tras el contacto inicial con los entrenadores (vía e-mail, teléfono y/o redes sociales) se concretó un lugar y horario para la entrevista de tal modo que no interfiriese en sus actividades profesionales, permitiendo así una atención exclusiva al investigador. Las entrevistas se almacenaron íntegramente en un dispositivo MP3 para poder realizar, posteriormente, las labores de transcripción, tabulación y análisis. La transcripción se comenzó el mismo día de la entrevista, para que estuviera lo más reciente posible para el investigador (Oliver, Serovich y Mason, 2005). Todas las transcripciones fueron enviadas a los entrenadores, en un intento de validar los respectivos contenidos y posibilitar las modificaciones que estimaran necesarias.

Para la tabulación y el análisis de los discursos se utilizó el método del Discurso del Sujeto Colectivo (DSC) (Lefèvre y Lefèvre, 2012), que permite representar el pensamiento de un colectivo a partir de la suma de extractos continuos y/o discontinuos de cada discurso individual, manteniendo la coherencia con cada parte que lo compone (Lefèvre y Lefèvre, 2012). El DSC se basa en la Teoría de las Representaciones Sociales ampliamente descrita por Serge Moscovici, que "se ocupa fundamentalmente de la interrelación entre sujeto y objeto y como se da el proceso de construcción del conocimiento, al mismo tiempo individual y colectivo" (Crusoé, 2004, p.106).

El DSC está constituido, por tanto, por tres figuras metodológicas: las ideas centrales (IC: descripción fidedigna del sentido de un discurso sobre un tema); las expresiones clave (ECH: transcripciones literales de extractos del discurso, que revelan la esencia de las IC); y el propio Discurso del Sujeto Colectivo (DSC: escrito en primera persona, es un discursosíntesis basado en el conjunto de ECH con la misma IC) (Lefèvre y Lefèvre, 2012).

El análisis de los discursos, se sustenta en el conocimiento proveniente de las impresiones e ideas de los entrenadores, que pudieran poner de manifiesto aspectos relevantes de lo que ese grupo piensa sobre las situaciones investigadas (Crusoé, 2004). Después, se identificaron las concepciones de los entrenadores sobre posibles similitudes y diferencias entre las categorías estudiadas (júnior y sénior) y como podría contribuir a identificar aspectos relacionados con el concepto de entrenamiento.

En la sección "Resultados" serán presentados los DSC con las procedencias de las entrevistas representadas de forma sobrescrita.

\section{Resultados}

El discurso de los entrenadores reveló inicialmente que las características de las categorías júnior y sénior son similares, realizándose incluso entrenamientos de forma conjunta. En este contexto, se observa la opinión unánime de los entrenadores sobre la especialización de las jugadoras y la mayor búsqueda de resultados cuando comparan la categoría junior con la sénior. Este posicionamiento constituyó el DSC1 (pautado en la IC-1: "Características de las categorías junior y sénior"):

\section{DSC1: Características de las categorías junior y sénior}

En júnior creo que mantienen la idea del juvenil aunque más específico ${ }^{S 2}$. No hago tanta diferencia de júnior a

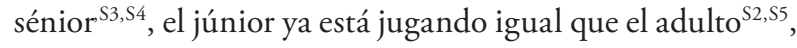
tiene que saber todo, pero es un camino ${ }^{52, S 3}$ y sirve para

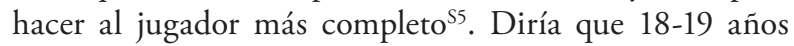
es la misma edad, cambiaría tal vez la exigencia de rotar el máximo posible a las chicas, muchos cambios, todo el mundo tendría por lo menos 10-12-15 minutos para ju$\operatorname{gar}^{\mathrm{S} 1}$. El entrenador tiene que comenzar a obtener resultados, puede hasta tener una paciencia mayor con los que están en el primer o segundo año de junior, pero ya tiene que empezar a obtener resultados de lo que entrenó toda la vida ${ }^{55}$. Es la categoría en la que tú vas a jugar y vas a hacer esto, esto y esto; y hay cosas que hay que prohibirles hacer, porque ahí ya es una competición para ganar el mismo partido ${ }^{55}$; el jugador tiene que demostrar rendimiento, tiene que ser efectivo ${ }^{\$ 4}$; tiene que tener autonomía para saber lo que está haciendo, la lectura ya tiene que ser automática ${ }^{\mathrm{S3}}$. La diferencia entre júnior y sénior es esa, júnior ejecuta, adulto sabe ejecutar, más allá de la cantidad/bagaje del juego ${ }^{S 3}$; el resultado pasa a estar en el primer lugar ${ }^{\mathrm{S}}$. En el adulto se tendrá en cuenta el proceso de aprendizaje, tiene que estar teóricamente completo, con todos los fundamentos dominados ${ }^{52}$, tener la lectura de todo, de todo lo que se ofrece ${ }^{S 3}$, con especialización total en los puestos ${ }^{S 1}$, en una o 2 posiciones $^{\mathrm{S2}}$. Mayor exigencia de la capacidad de resistencia, fuerza, biotipológica: el pívot cada vez más fuerte, la chica que marca de primera es cada vez más ve$\mathrm{loz}^{\mathrm{S1}}$. En júnior se trabaja mucho físico ${ }^{\mathrm{S2}}$, es un jugador más rápido porque está priorizando la parte física, y se trabaja mucho la parte física dejando la inteligencia de medio lado $^{\mathrm{S5}}$. En el adulto, ya se va más a la táctica ${ }^{\mathrm{S5}}$, aumenta la especificidad $^{\mathrm{S2}}$, todo el mundo tiene sus posiciones definidas ${ }^{56}$ y si no puedes manejar las instrucciones, estás fuera del equipo ${ }^{\text {S5. }}$.

El escenario representado en el DSC1 se desdobla hacia la preferencia por los métodos de enseńanza adoptados por los entrenadores en los entrenamientos: a) método tradicional (técnico - DSC2); b) situaciones de juego (DSC3); utilización de juegos, sin la connotación de entrenamiento (DSC4). Los discursos se presentan en la tabla 2 . 
Tabla 2: DSC elaborados a partir de la opinión de los entrenadores sobre aspectos específicos del entrenamiento

\section{DSC2: Abordaje técnico (S2, S3, S4, S5, S6)}

Mejora de la técnica, que considero que no tiene uno aprendido, mejora de los movimiento y respuestas que pueden suceder durante el juego ${ }^{54}$, tales como marcaje, contacto físico fuerte y posicionamiento ${ }^{55}$. Hay una mayor observación del gesto técnico ${ }^{54}$ con velocidad $^{\$ 3}$. Yo siempre vuelvo a esto: hago pase, salto de hacer pases en larga distancia, lanzar la pelota como en un contraataque ${ }^{\mathrm{S3}}$. Trabajo individualizado específico en las posiciones, especialistas en las posiciones, trabajo de técnica intensivo y sumado al colectivo; de ahí va a sumar todo ese trabajo al colectivo y montar el sistema ${ }^{\mathrm{s} 6}$. Creo que por lo menos el 50\% de su entrenamiento tiene que ser trabajo individualizado, si no el atleta acaba olvidando algunos movimientos, algunas cosas que no eran para perfeccionar allí y no consigue ejecutar ${ }^{\mathrm{s} 6}$.

Hacer que las acciones tácticas sean conocidas por todos, todos tienen que conocer las acciones tácticas, jugadas, movimientos, porque la gente comienza la jugada y el niño no sabe lo que tiene que hacer con los adultos ${ }^{\mathrm{s}}$.

\section{DSC3: Situaciones de juego $(S 1, S 2)$}

Me gusta trabajar con situaciones de juego ${ }^{\mathrm{S1}, \mathrm{S2}}: 4 \times 33$, 3×2 e $5 \times 4$ siempre me dará un pase de cara, un lanzamiento, una finta, es decir, ya está involucrado en esa acción lanzando con presión, pasando con presión, siempre con estímulos del juego ${ }^{\$ 1}$.

\section{DSC4: Juegos sin connotación de entrenamiento (S1, S2, S3)}

El juego pre-deportivo pasa a ser solo motivacional en el adulto, no porque ya no tenga esa función, sino porque ya no tiene la función de aprendizaje ${ }^{\mathrm{S2}}$. "Brinco de bobinho" ${ }^{33} \mathrm{y}$ cada semana tiene que tener un poco de fútbol, a ellas les gusta, es nuestra cultura, por lo menos 10 minutos, después de todo, ayuda a tener una movilidad de pierna fantástica, la chica que juega bien al fútbol defiende maravillosamente bien $^{\text {s1 }}$.

\section{Discusión}

Los entrenadores entrevistados desarrollan sus entrenamientos a partir de iniciativas vinculadas a las prefecturas municipales, y algunos también cuentan con apoyo de empresas privadas, especialmente por medio del ofrecimiento de servicios (como becas de estudios) o de colaboración financiera para costear dietas de alimentación, vivienda y transporte de las jugadoras. Los entrenamientos de los equipos de todos los entrevistados se llevan a cabo principalmente en gimnasios municipales, a excepción del equipo de S2, que también utilizaba un gimnasio perteneciente a un club.

Inicialmente los entrenadores abordaron las diferencias y similitudes entre las categorías júnior y sénior. El DSC1 evidenció que éstas se desarrollan en el mismo contexto de entrenamiento (con los mismos ejercicios) y de competición. Así, cabe señalar una división formal entre los equipos de competiciones solamente en la categoría júnior, de la que gran parte de las jugadoras de esa categoría componen la plantilla adulta.

Fijándonos en el número reducido de jugadoras y en la necesidad de presentar resultados explícitos para mantener sus apoyos y posibles patrocinadores, los entrenadores consideran imprescindible el trabajo similar en ambas categorías. El apuntamiento del DSC1 encuentra respaldo en las características presentadas en la etapa de inversión y posterior manutención en el deporte profesional (Côtè et al., 2007; Durand-Bush y Salmela, 2002), como la participación en un número mayor de competiciones organizadas y el aumento de la búsqueda de resultados.

En la etapa de inversión se espera que la jugadora se aproxime cada vez más al mejor nivel de rendimiento en la modalidad, y a partir de la intensificación de los entrenamientos, del aumento de la práctica planificada y de la participación y obtención de resultados en diferentes competiciones, pueden participar en el deporte profesional. (Bompa y Haff, 2009) muestran que jugadores de balonmano llegan a su pico de rendimiento sobre los 22-26 ańos. Hasta alcanzar la franja sugerida por los autores, las jugadoras necesariamente pasan en la etapa de inversión y manutención, por tres categorías (juvenil, junior y sénior), las cuales requieren diferentes particularidades y niveles de comprensión del juego.

De esta forma, se busca la excelencia por la consolidación del proceso de enseńanza-aprendizaje, principalmente por el hecho de que las jugadoras de categoría júnior hayan vivenciado diversas situaciones en los entrenamientos y en las competiciones de categorías anteriores (Menezes, Reis y Tourinho Filho, 2015), que proporcionarán la mejora de las capacidades técnicas, tácticas y motoras en las categorías que la preceden (Antón García, 1990). Por otro lado, las sesiones de entrenamiento se realizan en conjunto por el hecho de que algunas jugadoras de esas categorías trabajasen y/o estudiasen a lo largo de la semana, reduciendo la posibilidad de realizar los entrenamientos con el equipo completo; o por tener que dedicarse a otras tareas coincidentes con el mundo deportivo, no abordadas en el presente estudio. 
Estos apuntes suscitan el debate sobre la adecuación de procedimientos pedagógicos que respeten la heterogeneidad del equipo, muchas veces compuesto por adultos con larga experiencia y por jóvenes provenientes de las categorías de formación, aunque habituándose al aumento de la exigencia (competitiva y de comprensión del juego) en balonmano. Côtè, Erickson y Abernethy (2013) mencionan que el juego deliberado y la práctica espontánea generalmente se desarrollan en un contexto de edades mixtas, lo que expone a los niños y niñas más jóvenes a habilidades más avanzadas, para las cuales se tienen que esforzar, a la vez que las jóvenes de más edad sirven como soporte para el aprendizaje de las más pequeñas.

Para el contexto heterogéneo mencionado en el DSC1, aunque involucra la práctica deliberada y los diferentes enfoques de la enseńanza propuestos por los entrenadores, tales conceptos pueden ser asumidos por el hecho de que las jugadoras más jóvenes (como las que entran en la categoría junior), son expuestas a un juego probablemente más complejo y más intenso, mientras que las más experimentadas actúan como facilitadoras para el aumento del rendimiento de las jóvenes. En este sentido, el papel atribuido a las compañeras de equipo se basa en la formación de un grupo de amigas (Côtè, Baker y Abernethy, 2003) que también ayudarán a mantener un ambiente propicio para la mejora del balonmano.

Estas premisas justifican la importancia de las vivencias en las competiciones en diferentes grupos de edad, para que el entrenador pueda establecer criterios de enseńanza-aprendizaje para las jugadoras, culminando en un posicionamiento más paciente con ellas en el inicio de la categoría júnior. Los entrenadores destacan, por tanto, la cantidad de juegos como una diferencia importante entre tales categorías, por promover experiencias variadas a las jugadoras $y$, en cierto modo, las aproxima gradualmente a los requisitos de la categoría sénior. La categoría júnior ya forma parte de la etapa de entrenamiento de alto nivel, en la cual se dedica más tiempo a los contenidos específicos del balonmano (Ehret et al., 2002) y en esto se basa el papel del entrenador como mediador y motivador en los entrenamientos, especialmente por cambiar sus exigencias, que pueden justificar un mayor atractivo a los enfoques que se aproximan más a los requisitos específicos del balonmano.

El DSC1 destacó diferencias como la cantidad de juegos, la obtención de resultados y la ejecución de gestos técnicos, revelando que en estas categorías se produce un aumento de la búsqueda de resultados, principalmente por entender que se trata de etapas en las cuales existe una necesidad de ganar $\mathrm{y}$, al mismo tiempo, se consolidan las vivencias que las jugadoras tuvieron a lo largo de un proceso de enseñanza-aprendizaje, tal y como plantean otros autores (Baratti y Casali, 2008; Bompa y Haff, 2009; Ehret et al., 2002). Se entiende, por lo tanto, que se produce un aumento de la búsqueda de resultados, con mayor tolerancia a los errores cometidos en la categoría júnior en comparación con la sénior (mencionado en el DSC1), cuyo objetivo pasa a ser exclusivamente el de ganar partidos y competiciones.

Se pone de manifiesto el aumento de la especificidad en categoría júnior comparada con la categoría juvenil y, más aún, con la categoría sénior (en la cual las jugadoras ya deben tener puestos específicos definidos). Se espera, por tanto, que al ingresar en la categoría júnior, las jugadoras ya posean conocimientos especializados de los diferentes puestos específicos y sistemas (ofensivos y defensivos) de juego, principalmente porque muchas de ellas buscan una posición de consolidación en los equipos, para garantizar el acceso a beneficios como becas de estudios y ayudas económicas.

En el contexto heterogéneo presentado, materializado por la presencia de jugadoras con diferentes grupos de edad $y$ vivencias en el balonmano, la expectativa sobre los entrenamientos recae en el desarrollo de los más jóvenes en un ambiente más complejo y con mayor resultado. Tres posibilidades fueron presentadas por los entrenadores en la Tabla 2, siendo el método tradicional (DSC2) como el de mayor preferencia para ellos.

La preferencia por el método tradicional DSC2 se apoya en la mejora de la técnica (pase, recepción y lanzamientos, por ejemplo), y por eso puede estar directamente relacionado con la especialización deportiva que los entrenadores establecen como necesaria (DSC1). La importancia atribuida en el DSC2 a este método contrasta abruptamente con los desafíos propuestos para el entrenamiento de dos equipos constituidos por jugadoras con diferentes conocimientos y vivencias en el balonmano.

La premisa principal del método tradicional se basa en el aprendizaje/perfeccionamiento de la técnica en un contexto que no considera las complejas e imprevisibles relaciones de cooperación y de oposición del juego (Bunker y Thorpe, 1986; Galatti y Paes, 2007; Menezes, Marques y Nunomura, 2014; Santana, 2005) y por lo tanto, culmina en una baja transferencia entre el entrenamiento y el juego (Holt, Ward y Wallhead, 2006) y no contempla el contexto en el cual las acciones se desarrollan, en especial por ser abordadas de manera aislada al juego.

En el DSC2 se expone la necesidad de consolidación de jugadas ensayadas (de forma analítica), que se constituyen como una secuencia de movimientos prefabricados sin la oposición efectiva del adversario, que busca la reproducción de un comportamiento ofensivo con inicio, parte del medio y fin. Tal posicionamiento se orienta a las necesidades relatadas en el DSC1 en cuando a las diferencias y similitudes entre las categorías júnior y sénior, que requieren el desarrollo de acciones de acuerdo con las improvisaciones de los adversarios. La atención a niveles cada vez más elevados de conocimiento sobre el juego y de intervención en sus situaciones corrobora 
lo apuntado por (Feu Molina, 2006), en el sentido de que la jugadora juega como aprendió a jugar y, por basarse en el juego prefabricado, puede dejar de desarrollar sus acciones de manera libre y contextualizada.

Por otro lado, la preferencia presentada por las situaciones de juego (DSC3) se mostró secundaria. La posibilidad de desarrollar los aspectos tácticos, como lo propuesto en el método situacional, proporciona a los jugadores el desarrollo espacio-temporal, así como comprender la propia acción, la de los compañeros y de los adversarios (Greco, 2001). Su estructuración se realiza a partir de situaciones menores extraídas del juego que enfatizan aspectos relacionados directamente con las relaciones de cooperación y de oposición del juego, que exponen a las jugadoras a las presiones del adversario, del espacio, del tiempo y de resultados (con el objetivo del éxito en la secuencia ofensiva y/o defensiva).

La variabilidad de las situaciones de juego, la velocidad para percibir el ambiente y la selección de la decisión a tomar son aspectos predominantes para la actuación en un contexto de juego que varía dinámicamente (Menezes, 2012). Destaca la importancia de la capacidad de toma de decisión y de resolución de problemas por los jugadores (Gréhaigne y Godbout, 1995; Matias y Greco, 2010), especialmente considerando la perspectiva apuntada en el DSC1, orientación que concuerda con el DSC3, aunque se orienta contrariamente al DSC2.

Llama la atención también el posicionamiento del DSC4 sobre los juegos para que sean utilizados apenas como calentamiento, como un momento de relajación en el entrenamiento, sin la finalidad de desarrollar los aspectos tácticos de las jugadoras y el modelo de juego en equipo. Esa posición excluye la importancia del contexto complejo que los juegos pueden presentar a las jugadoras, principalmente relacionadas con la percepción del ambiente del juego, con la elaboración de respuestas y con la toma de decisiones en ambientes dinámicos y complejos.

La enseñanza por medio de juegos (pautado en la corriente global-funcional) se caracteriza por la presencia de situaciones-problema pautadas en juegos de menor complejidad, que envuelven los elementos presentes en la lógica del juego (Galatti y Paes, 2007; Menezes et al., 2014), poseen carácter lúdico y son adecuados para los practicantes (Galatti y Paes, 2007), lo que los convierte en motivantes para el contexto del entrenamiento, principalmente por responder al ansia de jugar.

Tanto el método situacional como la enseñanza por medio de juegos exige a las jugadoras la resolución de situacionesproblema que el juego va a ofrecer a partir de las interacciones entre ellas, cuyas situaciones complejas de entrenamiento pueden enfatizar comportamientos técnicos o tácticos específicos a partir de contextos con demandas transferibles al juego formal. Parece ser este el punto central extraído del DSC1, ya que para aumentar la obtención de resultados, por mejor comprensión del juego y por niveles de excelencia cada vez mayores, es necesario desarrollar la capacidad de toma de decisiones de las jugadoras.

En la perspectiva apuntada por los entrenadores, el juego no asume su papel indispensable para desarrollar y consolidar diversos conceptos técnico-tácticos en las sesiones de entrenamiento, ni su papel de motivador del grupo de jugadoras. Esta constatación revela la dificultad de comprender las virtudes de la enseñanza por medio de juego para los equipos de las categorías estudiadas, que puede estar relacionada con las posibles experiencias de los entrenadores en la progresión, cuyas vivencias enfatizan el aspecto tecnocrático de la enseñanza (Modolo, Madeira, Santos, D’Almeida y Menezes, 2017) y a la escasez de iniciativas de diferentes entidades que regulan el balonmano (Musa, Modolo, Barreira, Tsuji y Menezes, 2017), lo que fortalece el paradigma que defiende que es necesario aprender la técnica para aprender a jugar. De este modo, el DSC4 revela apenas un vínculo con el carácter lúdico, que suena como sinónimo de "descompromiso" de la enseńanza por medio de juegos que puede estar asociado al hecho de no abordar explícitamente un "contenido", que puede estar fundamentado en sus procesos de formación como jugadores y entrenadores.

Tras analizar el proceso de enseñanza-aprendizaje del balonmano a largo plazo a partir de las perspectivas apuntadas en las categorías cadete y juvenil (Menezes, Ramos, Marques y Nunomura, 2018), se percibe un mantenimiento en las preferencias por los métodos de enseñanza. Especialmente en la categoría juvenil, inmediatamente anterior a la categoría júnior (Menezes et al., 2018), apuntan que los entrenadores priorizan la enseñanza por medio del método tradicional, seguido por las situaciones de juego y, por último, por los juegos (con una función relacionada al calentamiento o simplemente por diversión).

Se observa, por lo tanto, que a medida que se avanza hacia la categoría júnior existe una mayor tendencia a utilizar el método tradicional. Se cree que este cambio refleja una mayor valoración de las acciones de las jugadoras para mejorar parámetros como la eficacia y la eficiencia (con vistas al resultado), cuando comparadas con el aprendizaje de cuestiones que envuelven el contexto técnico-táctico y la toma de decisiones. El desajuste entre priorizar la técnica o aspectos técnico-tácticos del juego parece distanciar a los entrenadores de los objetivos mencionados en el DSC1, en especial a la mejora de la eficacia en el juego.

Los discursos revelaron que los entrenadores valoran la integración entre los equipos, por diferentes motivos, y que existe una mayor demanda del rendimiento. Sin embargo, las diferencias entre las categorías estudiadas se centran en las experiencias y el conocimiento de las jugadoras sobre el juego. Aunque los entrenadores han informado sobre la necesidad de obtener resultados por lo que han desarrollado en los juga- 
dores a lo largo del proceso de entrenamiento, aún se necesitan más experiencias relacionadas con el entorno competitivo para que se sientas seguros en la categoría sénior.

Se espera que a medida que los jugadores conozcan mejor el juego y posean experiencias diversificadas provenientes de las sesiones de entrenamiento y de las competiciones, sus interacciones pasarán a ser más complejas, surgiendo contextos en los cuales se enfrentarán a nuevos desafíos. Sin embargo, para contemplar esta perspectiva, es necesario utilizar en las sesiones de entrenamiento métodos que se aproximen a la resolución de las situaciones-problema, como el situacional y la enseñanza por medio de juegos para mejorar el repertorio motor y ampliar el repertorio cognitivo que respalda el proceso de toma de decisiones.

\section{Consideraciones finales}

Este estudio investigó la opinión de los entrenadores de balonmano del Estado de São Paulo en las categorías júnior y sénior femenino. A lo largo de la permanencia de las jugadoras en la categoría júnior, existe una mayor preocupación por los resultados competitivos (culminando en la categoría sénior) y la búsqueda constante de parámetros de eficacia que se transforman en resultados competitivos.

Este estudio avanza en la investigación de cómo se da el proceso de entrenamiento en las categorías júnior y sénior de balonmano femenino, posibilitando identificar los aspectos que envuelven la búsqueda por el rendimiento en dichas etapas, en especial de las características de esas categorías y de los procedimientos pedagógicos adoptados por los entrenadores. Los resultados de este estudio podrán ayudar en la identificación de un contexto en el cual el balonmano se desarrolle en diferentes contextos, así como apoyar una posible propuesta de proceso de enseñanza-aprendizaje, a través de la ampliación de reflexiones sobre el proceso de formación de jugadoras de balonmano, especialmente en las categorías que preceden a la sénior.

Se realizó el análisis de un escenario importante del Estado de Sáo Paulo con respecto a la enseñanza del balonmano en equipos representativos que generalmente consisten en jugadores con características heterogéneas. Se identificaron dos limitaciones en este estudio. La primera se refiere al hecho de analizar un grupo de entrenadores de equipos femeninos del Estado de Sáo Paulo, motivo por el cual los resultados pueden no ser generalizables a entrenadores de otras regiones del Estado, de otros estados, de otros países o de equipos masculinos. La segunda limitación está relacionada con el distanciamiento entre el discurso de los entrenadores y su práctica.

Como futuras perspectivas de estudio destacan la posibilidad de ampliación del número de entrenadores entrevistados, la participación de los entrenadores de equipos de sexo masculino y de entrenadores de otros Estados y otros países.

\section{Referencias}

1. Antón García, J. L. (1990). Balonmano: fundamentos y etapas de aprendizaje. Madrid: Gymnos Editorial.

2. Baratti, A., \& Casali, E. (2008). Balonmano: desarrollo de los fundamentos por médio de ejercitaciones y formas jugadas. Armenia: Editorial Kinesis.

3. Bompa, T. O., \& Haff, G. G. (2009). Periodization: theory and methodology of training. Champaign: Human Kinetics.

4. Bunker, B., \& Thorpe, R. (1986). The curriculum model. In R. Thorpe, D. Bunker, \& L. Almond (Eds.), Rethinking games teaching (pp. 7-10). Loughborough: University of Technology.

5. Crusoé, N. M. d. C. (2004). A Teoria das Representaçôes Sociais em Moscovici e sua importância para a pesquisa em educação. Aprender: Caderno de Filosofia e Psicologia da Educação, II(2), 105-114.

6. Côtè, J., Baker, J., \& Abernethy, B. (2003). From play to practice: a developmental framework for the acquisition of expertise in team sport. In J. Starkes \& K. Ericsson (Eds.), Expert performance in sports: advances in research on sport expertise (pp. 89-113). Champaign, IL: Human Kinetics.

7. Côtè, J., Baker, J., \& Abernethy, B. (2007). Practice and play in the development of sport expertise. In R. Eklund \& G. Tenenbaum (Eds.), Handbook of sport psychology (pp. 184-202). Hoboken: Wiley.

8. Côtè, J., Erickson, K., \& Abernethy, B. (2013). Play and practice during childhood. In J. Côtè \& R. Lidor (Eds.), Conditions of children's talent development in sport (pp. 9-20). Morgantown, WV: Fitness Information Technology.

9. Durand-Bush, N., \& Salmela, J. H. (2002). The development and maintenance of expert athletic performance: perceptions of wolrd and olympic champions. Journal of Applied Sport Psychology, 14(3), 154-171.

10. Ehret, A., Späte, D., Schubert, R., \& Roth, K. (2002). Manual de handebol: treinamento de base para crianças e adolescentes. São Paulo: Phorte Editora.

11. Feu Molina, S. (2006). Organización didáctica del proceso de enseñanza-Aprendizaje para la construcción del juego ofensivo en balonmano. E-Balonmano, 2(4), 53-66.

12. Galatti, L. R., \& Paes, R. R. (2007). Pedagogia do esporte e a aplicação das teorias acerca dos jogos esportivos coletivos em escolas de esportes: o caso de um clube privado de Campinas-SP. Conexóes, 5(2), 31-44.

13. Galatti, L. R., Reverdito, R. S., Scaglia, A. J., Paes, R. R., \& Seoane, A. M. (2014). Pedagogia do esporte: tensão na ciência e o ensino dos jogos esportivos coletivos [Sport pedagogy: tension in science and teaching of collective sports games]. Revista da Educação Física/UEM, 25(1), 153-162.

14. Greco, P. J. (2001). Métodos de ensino-aprendizagem-treinamento nos jogos esportivos coletivos. In E. Garcia \& K. Lemos (Eds.), Temas Atuais VI em Educação Física e Esportes (pp. 48-72). Belo Horizonte: Editora Health.

15. Greco, P. J., Silva, S. A., \& Greco, F. L. (2012). O sistema de formação e treinamento esportivo no handebol brasileiro (SFTE-HB). In P. J. Greco \& J. J. Fernández Romero (Eds.), Manual de handebol: da iniciação ao alto nivel (pp. 235-250). São Paulo: Phorte.

16. Gréhaigne, J.-F., \& Godbout, P. (1995). Tactical knowledge in team sports from a constructivist and cognitivist perspective. Quest, 47, 490505.

17. Holt, J. E., Ward, P., \& Wallhead, T. L. (2006). The transfer of learning from play practices to game play in young adult soccer players. Physical Education and Sport Pedagogy, 11(2), 101-118. doi:10.1080/17408980600708270 
18. Lefèvre, F., \& Lefèvre, A. M. C. (2012). Pesquisa de representação social: um enfoque qualiquantitativo (2 ed.). Brasília: Liber Livro Editora.

19. Marconi, M. d. A., \& Lakatos, E. M. (2011). Metodologia cientifica (6 ed.). São Paulo: Atlas.

20. Matias, C. J. A. d. S., \& Greco, P. J. (2010). Cognição e ação nos jogos esportivos coletivos. Ciência \& Cognição, 15(1), 252-271.

21. Menezes, R., Ramos, N., Marques, R., \& Nunomura, M. (2018). Teaching handball to U-16 and U-18 women's teams: coaches' perspective on the long-term. Motriz, 24(4), e101838.

22. Menezes, R. P. (2012). Contribuições da concepção dos fenômenos complexos para o ensino dos esportes coletivos. Motriz, 18(1), 34-41.

23. Menezes, R. P., Marques, R. F. R., \& Morato, M. P. (2016). Handball coaches' perception of the offensive and defensive variables of the game in u-12 teams. Motricidade, 12(3), 6-19. doi:10.6063/motricidade.4581

24. Menezes, R. P., Marques, R. F. R., \& Nunomura, M. (2014). Especialização esportiva precoce e o ensino dos jogos coletivos de invasão. Movimento, 20(1), 351-373.

25. Menezes, R. P., Marques, R. F. R., \& Nunomura, M. (2015). O ensino do handebol na categoria infantil a partir dos discursos de treinadores experientes [Handball teaching in under-14 teams according to experienced coaches' discourses]. Movimento, 21(2), 463-477.

26. Menezes, R. P., Marques, R. F. R., \& Nunomura, M. (2017). Teaching handball to players under-12: the perspective of Brazilian coaches. $M o$ triz, 23(4).
27. Menezes, R. P., Reis, H. H. B. d., \& Tourinho Filho, H. (2015). Ensino-aprendizagem-treinamento dos elementos técnico-táticos defensivos individuais do handebol nas categorias infantil, cadete e juvenil [Teaching-learning-training of individual technical-tactical elements of handball for under-14, under-16 and under-18 teams]. Movimento, 21(1), 261-273

28. Modolo, F., Madeira, M. G., Santos, W. R., D’Almeida, M. d. P., \& Menezes, R. P. (2017). Contextos e situaçóes de aprendizagem de treinadores de handebol em âmbito escolar de um município do Estado de São Paulo. Movimento, 23(4), 1203-1216.

29. Musa, V., Modolo, F., Barreira, C., Tsuji, G., \& Menezes, R. P. (2017) Representaçốes dos treinadores sobre o papel das instituiçốes reguladoras do handebol para a sua formação. Revista Portuguesa de Ciências do Desporto, S1a, 298-306.

30. Oliver, D., Serovich, J., \& Mason, T. (2005). Constraints and opportunities with interview transcription: Towards reflection in qualitative research. Social Forces, 84(2), 1273-1289. doi:10.1353/ sof. 2006.0023

31. Santana, W. C. (2005). Pedagogia do esporte na infância e complexidade. In R. R. Paes \& H. F. Balbino (Eds.), Pedagogia do esporte: contextos e perspectivas (pp. 1-24). Rio de Janeiro: Guanabara Koogan.

32. Triviños, A. (1987). Introdução à pesquisa em ciências sociais: a pesquisa qualitativa em educação (1 ed.). São Paulo: Atlas. 